Check for updates

Cite this: Chem. Sci., 2019, 10, 9605

๑ All publication charges for this article have been paid for by the Royal Society of Chemistry

\section{Plasmon-promoted electrocatalytic water splitting on metal-semiconductor nanocomposites: the interfacial charge transfer and the real catalytic sites $\dagger$ t.}

\author{
Lili Du, Guodong Shi, Yaran Zhao, Xiang Chen, Hongming Sun, Fangming Liu, \\ Fangyi Cheng (D) and Wei Xie (DD*
}

\begin{abstract}
Plasmonic metal nanoparticles (NPs) have emerged as promising visible light harvesters to facilitate solarto-chemical energy conversion via the generation of hot electrons by non-radiative decay of plasmons. As one of the most promising renewable energy production methods for the future, electrocatalytic water splitting is an ideal chemical reaction in which plasmonic NPs can be utilized for direct solar-to-fuel conversion. Due to the rapid carrier recombination on plasmonic NPs, hybrid photocatalysts integrating metals and semiconductors are usually employed to separate the hot electrons and holes. However, an understanding of the catalytic mechanism, which is critical for rational design of plasmonic electrocatalysts, including the interfacial charge transfer pathway and real reactive sites, has been lacking. Herein, we report on the combination of plasmonic Au NPs and semiconductors (Ni and/or Co hydroxides) for plasmon-promoted electrocatalytic water splitting. By using surface-enhanced Raman spectroscopy (SERS), we find a strong spontaneous interfacial charge transfer between $\mathrm{Au}$ and $\mathrm{NiCO}$ layered double hydroxide (LDH), which facilitates both the oxygen and hydrogen evolution reactions. The real catalytic sites on the hybrid material are confirmed by selective blocking of the metal surface with a thiol molecular monolayer. It is found that the plasmon-promoted oxygen evolution occurs on the LDH semiconductor but surprisingly, the hydrogen evolution sites are mainly located on the Au NP surface. This work demonstrates the critical role of interfacial charge transfer in hot electron-driven water splitting and paves the way for rational design of high-performance plasmonic electrocatalysts.
\end{abstract}

Received 6th July 2019

Accepted 28th August 2019

DOI: $10.1039 / c 9 s c 03360 b$

rsc.li/chemical-science evolution reaction, HER) half-reactions is one of the most promising clean and renewable energy production methods for the future. ${ }^{\mathbf{1 7 - 2 0}}$ It is therefore an ideal chemical reaction for the application of plasmonic NPs, in which solar energy can be directly converted to hydrogen fuel. ${ }^{21}$ Because of the rapid charge-carrier recombination and the intrinsically low electrocatalytic activity of plasmonic NPs, the plasmonic NPs were usually employed together with conventional semiconductor catalysts to separate the charge carriers. ${ }^{2,23} \mathrm{Au}$ NP-decorated $\mathrm{Ni}(\mathrm{OH})_{2}$ nanosheets and $\mathrm{Au}$ nanorod-decorated $\mathrm{MoS}_{2}$ nanosheets represent the few examples of plasmonic metal-semiconductor electrocatalysts for the OER and HER, respectively. ${ }^{\mathbf{2 4 , 2 5}}$ In oxygen production, the hot holes facilitate electron transfer from a conventional semiconductor catalyst to plasmonic metal NPs and thus enhance the water oxidation on the semiconductor surface. In contrast, the hot electrons transferred from the metal to the semiconductor were thought to be responsible for the plasmon-promoted hydrogen production. Generally, the semiconductors were believed to be the catalytically active sites for both oxidation and reduction of water. However, experimental evidence of the real catalytic site
Key Lab of Advanced Energy Materials Chemistry (Ministry of Education), Renewable Energy Conversion and Storage Center, College of Chemistry, Nankai University, Weijin Rd. 94, Tianjin 300071, China. E-mail: wei.xie@nankai.edu.cn $\dagger$ Dedicated to the 100th anniversary of Nankai University.

\$ Electronic supplementary information (ESI) available. See DOI: 10.1039/c9sc03360b 
on the hybrid surfaces has been lacking. To design high performance plasmonic electrocatalysts for visible lightassisted water splitting, it is important to know how the hot carriers are transferred and where the catalytic sites of the reactions are.

Besides the hot carriers, plasmonic metal NPs also provide localized electromagnetic fields that enable surface-enhanced Raman spectroscopic (SERS) detection of adsorbed chemical species. $^{26,27}$ In the past decade, SERS has been proved to be a powerful analytical technique for interfacial characterization. ${ }^{28}$ Because of the high sensitivity, surface selectivity and fingerprint specificity, SERS is very sensitive to the electronic properties of the metal surface and therefore promising for investigating the interfacial charge transfer to understand the mechanism of plasmon-promoted electrocatalysis. ${ }^{29,30}$

In this study, we prepared $\mathrm{Au} \mathrm{NP}$-decorated $\mathrm{Co}(\mathrm{OH})_{2}$, $\mathrm{Ni}(\mathrm{OH})_{2}$ and $\mathrm{Ni}-\mathrm{Co}$ layered double hydroxide $\left(\mathrm{Au} / \mathrm{Co}(\mathrm{OH})_{2}, \mathrm{Au} /\right.$ $\mathrm{Ni}(\mathrm{OH})_{2}$ and $\left.\mathrm{Au} / \mathrm{NiCo} \mathrm{LDH}\right)$ as plasmonic electrocatalysts for water splitting under visible light irradiation. The hydroxide semiconductors are conventional water splitting electrocatalysts, while the Au NPs with stable chemical properties and strong plasmonic activity act as visible light harvesters. ${ }^{31-33}$ Before the water splitting experiments, we employed SERS to study the interfacial charge transfer between the hydroxide semiconductors and the decorated $\mathrm{Au}$ NPs in a semiconductor-metal-molecule hybrid system, where 2,6-dimethylphenyl isocyanide (DMPIC) was used as a probe molecule. DMPIC interacts with the metal surface via orbital hybridization and the strength of the $\mathrm{N} \equiv \mathrm{C}$ bond is affected by the electron abundance of the SERS substrates (Au NPs). ${ }^{34}$ According to the SERS spectra, we can determine if the plasmonic Au NPs lose or gain electrons after immobilizing them on the different hydroxides.

Finding out the real catalytic site on a hybrid surface is very challenging. The distinct $\mathrm{Au}$ and hydroxide surfaces offer the opportunity of selective coating of thiol molecules on the Au surface. After coating, although the semiconductor surface remains unchanged, the Au surface will not be available any more for the reactions. We incubated the Au/NiCo LDH hybrid material in a solution of thiophenol (TP) before the visible lightassisted electrocatalytic water splitting. Interestingly, a remarkably decreased catalytic activity of the Au/NiCo LDH hybrid material was detected in the HER, while no significant decrease was observed in the OER. It is clear that the main catalytic sites of the OER and HER are different. Based on the SERS study of the interfacial charge transfer between Au NPs and NiCo LDH, a reasonable mechanism of the plasmon-promoted electrocatalysis has been proposed. This new mechanism is different from the previously proposed ones: (1) the electron transfer in the plasmonic metal-semiconductor hybrid system is unidirectional, both in the OER and HER. (2) The HER takes place mainly on the plasmonic Au surface. The present study highlights the important role of the interfacial charge transfer between a plasmonic light harvester and a conventional electrocatalyst, and also identifies the real catalytic sites on the hybrid electrocatalyst for plasmon-promoted electrocatalytic water splitting.

\section{Experimental}

\section{Materials}

Chloroauric acid ( $\mathrm{HAuCl}_{4}$, Sigma-Aldrich), sodium borohydride $\left(\mathrm{NaBH}_{4} \text {, Sigma-Aldrich), cobalt acetate (Co(Ac) }\right)_{2}, 99 \%$, Aladdin), nickel acetate $\left(\mathrm{Ni}(\mathrm{Ac})_{2}, 99 \%\right.$, Aladdin), 2,6-dimethylphenyl isocyanide (98\%, Energy-Chemical), methanol (Tianjin Kangkede Technology Co., Ltd.), potassium hydroxide (KOH, 97\%, Aladdin), and Ni foam (Innochem) were used without further purification.

\section{Synthesis of NiCo $\mathrm{LDH}, \mathrm{Ni}(\mathrm{OH})_{2}$ and $\mathrm{Co}(\mathrm{OH})_{2}$}

NiCo LDH was prepared through a modified solvothermal method..$^{35} \mathrm{Ni}(\mathrm{Ac})_{2} \cdot 4 \mathrm{H}_{2} \mathrm{O}(1 \mathrm{mmol})$ and $\mathrm{Co}(\mathrm{Ac})_{2} \cdot 4 \mathrm{H}_{2} \mathrm{O}(0.5$ $\mathrm{mmol}$ ) were dissolved together in $15 \mathrm{~mL}$ methanol. After stirring for $30 \mathrm{~min}$, the mixture was transferred into a $50 \mathrm{~mL}$ Teflon-lined autoclave with a piece of treated $\mathrm{Ni}$ foam inside. The autoclave was then sealed and kept in an oven at $180^{\circ} \mathrm{C}$ for 12 hours and naturally cooled down to room temperature. The substrate was washed several times with deionized water/ ethanol and dried in an oven at $60{ }^{\circ} \mathrm{C}$ for 2 hours. The catalyst loading on the $\mathrm{Ni}$ foam was determined to be $\sim 1.4 \mathrm{mg}$ $\mathrm{cm}^{-2}$. A powdery sample was prepared using the same method, just without the presence of $\mathrm{Ni}$ foam. $\mathrm{Ni}(\mathrm{OH})_{2}$ and $\mathrm{Co}(\mathrm{OH})_{2}$ were prepared using similar methods.

\section{Synthesis of Au NPs}

According to a previous study, ${ }^{36} \mathrm{Au}$ nanoparticles were synthesized by $\mathrm{NaBH}_{4}(0.1 \mathrm{M})$ reduction of $\mathrm{HAuCl}_{4}(0.25 \mathrm{mM})$. The color of the colloid is wine red, indicating the formation of plasmonic Au nanoparticles. Then, $1 \mathrm{~mL}$ toluene was added to a Petri dish containing $5 \mathrm{~mL}$ colloid of Au NPs. Subsequently, $0.5 \mathrm{~mL}$ dimethylformamide (DMF) was added dropwise. After the evaporation of methylbenzene, a compact Au NP film was formed at the water/oil interface. The film was then transferred gently to a piece of $\mathrm{Ni}$ foam to form the $\mathrm{Au}$ NPs/Ni foam electrode. ${ }^{37}$

\section{Synthesis of $\mathrm{Au} / \mathrm{NiCo} \mathrm{LDH}, \mathrm{Au} / \mathrm{Ni}(\mathrm{OH})_{2}$, and $\mathrm{Au} / \mathrm{Co}(\mathrm{OH})_{2}$}

NiCo LDH was decorated with Au NPs via electrostatic interactions. NiCo LDH supported on $\mathrm{Ni}$ foam was immersed into a colloidal suspension of Au NPs and shaken slightly until the colloid became colorless. After complete immobilization of the Au NPs on the NiCo LDH, the Au/NiCo LDH nanocomposites were dried in air. For comparison, 5, 10 and $15 \mathrm{~mL}$ Au colloids were used and the $\mathrm{Au}$ mass percentage was determined to be 1.7, 3.3, and $5.1 \mathrm{wt} \%$, respectively. $\mathrm{Au} / \mathrm{Ni}(\mathrm{OH})_{2}$ and $\mathrm{Au} / \mathrm{Co}(\mathrm{OH})_{2}$ were prepared using the same method (10 mL Au colloid).

\section{Preparation of DMPIC-functionalized Au NP, Au/NiCo LDH, $\mathrm{Au} / \mathrm{Ni}(\mathrm{OH})_{2}$ and $\mathrm{Au} / \mathrm{Co}(\mathrm{OH})_{2}$ substrates for SERS measurements}

Powdery NiCo $\mathrm{LDH}, \mathrm{Ni}(\mathrm{OH})_{2}$ and $\mathrm{Co}(\mathrm{OH})_{2}$ were dispersed in $1 \mathrm{~mL} \mathrm{Au}$ colloid (60 nm). Subsequently, $10 \mu \mathrm{L}$ of $10 \mathrm{mM}$ ethanolic DMPIC solution was added. The mixture was incubated 
for $30 \mathrm{~min}$ to allow the formation of a molecular monolayer on the Au surface. The samples were then washed two times with ethanol and resuspended in $1 \mathrm{~mL}$ water. The prepared samples were directly used for SERS measurements.

\section{Characterization}

SEM images were obtained on a JEOL JSM-7500F electron microscope and high-resolution TEM was performed on a Tecnai G2 F20 S-TWIN at $200 \mathrm{kV}$. X-ray diffraction patterns were recorded on a Rigaku Mini Flex 600 powder diffractometer $(\mathrm{Cu}$ $\mathrm{K} \alpha$ radiation). The surface material valence states of the composite were analyzed using X-ray photoelectron spectroscopy (XPS) (Thermo Scientific ESCALAB 250Xi). The potential values of samples dispersed in water were determined on a Malvern Nano ZS90 Zetasizer. The extinction of the samples was measured using a UV-Vis spectrometer (PerkinElmer Lambda 750). The content of Au was determined by ICP-AES (PerkinElmer Optima 8300).

\section{SERS measurements}

The SERS spectra were acquired with a Horiba LabRAM HR Evolution Raman microscope with an excitation wavelength of $632.8 \mathrm{~nm}$ for all measurements. And the measurements under different potentials were performed by using a homemade twoelectrode configuration with a CHI-660E electrochemical workstation to control the applied potential.

\section{Photoelectrochemical (PEC) measurements}

PEC measurements were carried out on an electrochemical workstation (CHI660E, Shanghai, CH. Instruments, China) using a typical three electrode system, and a solar simulator (NBeT Solar-150 Inc.) applying AM 1.5G irradiation was employed as the light source $\left(100 \mathrm{~mW} \mathrm{~cm}^{-2}\right)$. Colored sources were supported by filters with different passing wavelengths. A graphite rod and standard $\mathrm{Hg} / \mathrm{HgO}$ electrode were used as the counter and reference electrodes, respectively, in an aqueous solution of $1 \mathrm{M} \mathrm{KOH}$. The OER catalytic activity was investigated using linear sweep voltammetry (LSV) at a scan rate of $1 \mathrm{mV} \mathrm{s}^{-1}$ in an $\mathrm{O}_{2}$-saturated solution of $\mathrm{KOH}$, while the HER tests were carried out using LSV at a sweep rate of $1 \mathrm{mV} \mathrm{s}^{-1}$ in a $\mathrm{H}_{2}$ saturated solution. The water splitting electrolysis was performed in $1 \mathrm{M} \mathrm{KOH}$ using a two-electrode configuration. All tested potentials $v s$. the $\mathrm{Hg} / \mathrm{HgO}$ electrode were converted to the RHE scale according to the Nernst equation $E_{\mathrm{RHE}}=E_{\mathrm{Hg} / \mathrm{HgO}}+$ $0.0591 \times \mathrm{pH}+0.098 \mathrm{~V}$. All polarization curves were reported with $i R$ compensation for ohmic losses: $E=E_{\mathrm{m}}-i R_{\mathrm{s}}$, where $E$ is the $i R$-corrected potential, $E_{\mathrm{m}}$ is the measured potential and $R_{\mathrm{s}}$ is the resistance of the solution. Electrochemical impedance spectra (EIS) were measured at an overpotential of $250 \mathrm{mV}$ from $0.1 \mathrm{~Hz}$ to $100 \mathrm{KHz}$.

\section{Results and discussion}

\section{Characterization of nanocomposites}

Fig. S1 shows the preparation process of Au/NiCo LDH. TEM images of the NiCo LDH prepared via a solvothermal method are shown in Fig. S2.\$ Au NPs with a size of $\sim 5.75 \mathrm{~nm}$ (Fig. S3\$) were prepared by $\mathrm{NaBH}_{4}$ reduction of $\mathrm{HAuCl}_{4}$. Because of the opposite interfacial charges (Fig. S4 $\$$ ), the plasmonic Au NPs were successfully loaded on the hydroxides via electrostatic interactions, forming the $\mathrm{Au} / \mathrm{NiCo} \mathrm{LDH}$ nanocomposites. The SEM/TEM images and the energy dispersive X-ray spectroscopy (EDS) mapping images shown in Fig. 1 reveal that the Au NPS were uniformly interspersed throughout the surface of NiCo LDH nanosheets. Using similar methods, Au NP-decorated $\mathrm{Co}(\mathrm{OH})_{2}$ and $\mathrm{Ni}(\mathrm{OH})_{2}$ were successfully prepared and the characterization results are shown in Fig. S5-S7+.

\section{Detection of the interfacial charge transfer by using SERS}

As a surface-sensitive molecular spectroscopic technique, SERS was used to investigate the interfacial charge transfer between the $\mathrm{Au}$ NPs and the conventional hydroxide electrocatalysts (semiconductor). The $\mathrm{N} \equiv \mathrm{C}$ symmetric stretching of DMPIC located within the spectral range of 2150 to $2200 \mathrm{~cm}^{-1}$ is very sensitive to the electronic structure of the metal surface. ${ }^{38}$ In this work, we used DMPIC as a molecular probe and compared the $\mathrm{N} \equiv \mathrm{C}$ stretching enhanced by Au NPs immobilized on the three different semiconductors: $\mathrm{Co}(\mathrm{OH})_{2}, \mathrm{Ni}(\mathrm{OH})_{2}$ and NiCo LDH. Fig. 2a illustrates the $\mathrm{N} \equiv \mathrm{C}$ band shift due to the metalmolecule electron transfer. The DMPIC molecule interacts with the Au surface via the $\sigma$ lone electron pair and unoccupied $\pi^{*}$ frontier orbitals of $\mathrm{N} \equiv \mathrm{C}$, which hybridize with the d orbitals of the metal to form $\sigma-d$ donation and $d-\pi^{*}$ back donation. ${ }^{39}$ Electron transfer from the support material to the metal decreases the $\sigma-d$ interaction but enhances the $d-\pi^{*}$ backdonation; this weakens the $\mathrm{N} \equiv \mathrm{C}$ bond strength and leads to a red-shift of the SERS band. ${ }^{40}$ To verify the relation between the band shift and the interfacial charge transfer, we constructed a two-electrode system and the DMPIC modified Au NPs were immobilized on the working electrode. As shown in Fig. 2b, under an external voltage $(-0.4$ to $0 \mathrm{~V})$, the SERS band of $\mathrm{N} \equiv \mathrm{C}$ red-shifts. In contrast, a blue-shift of the band is observed on

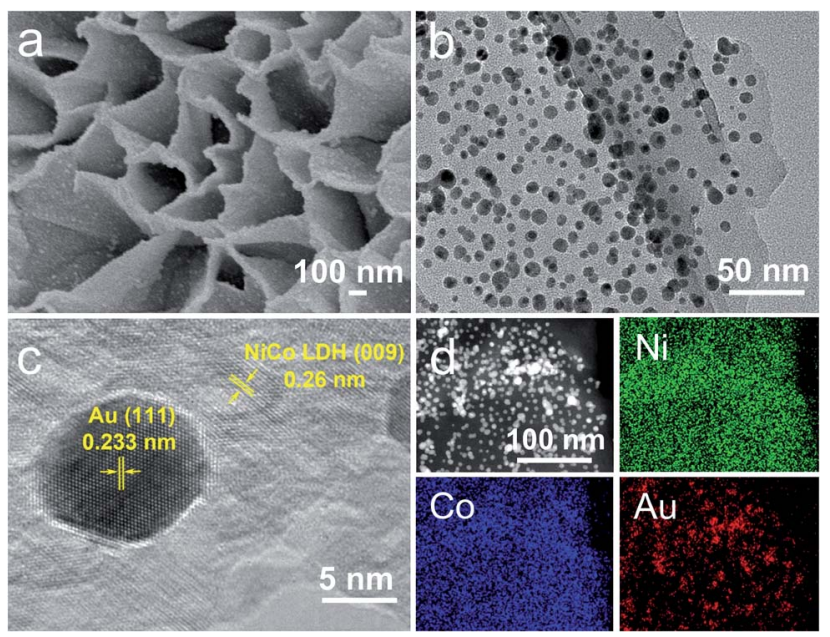

Fig. 1 Characterization of the Au/NiCo LDH. SEM (a), TEM (b), highresolution TEM (c), and EDS elemental mapping images (d) of the Au/ NiCo LDH nanocomposite. 
a
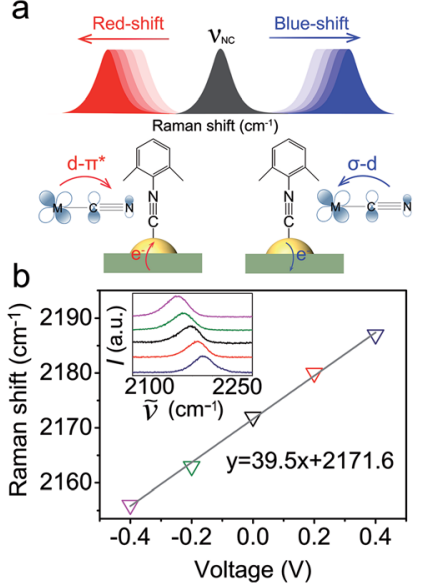

C

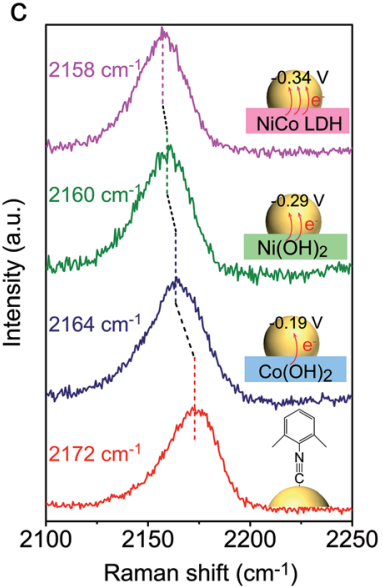

a

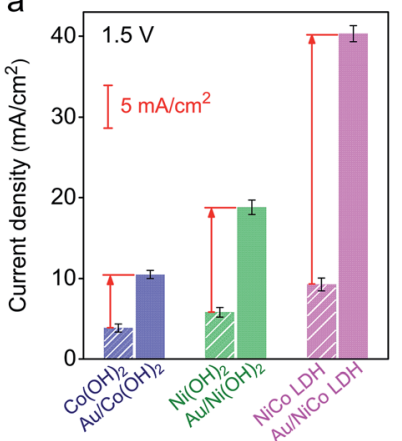

b

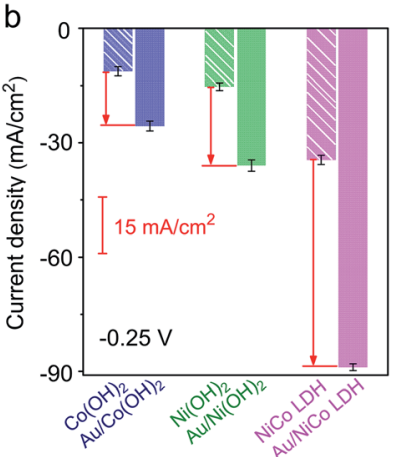

Fig. 3 Electrochemical performance before and after decoration of $\mathrm{Au}$ NPs. Current densities of $\mathrm{Co}(\mathrm{OH})_{2}, \mathrm{Ni}(\mathrm{OH})_{2}$ and $\mathrm{NiCO} \mathrm{LDH}$ with (solid column) and without (striped column) Au NPs toward the OER (a) and HER (b).

Fig. 2 SERS detection of interfacial charge transfer between Au NPs and semiconductors. (a) Schematic illustration of the interfacial charge transfer in the semiconductor-metal-molecule (DMPIC) system and the corresponding SERS band shift. (b) Raman shift of the symmetric stretching mode of $\mathrm{N} \equiv \mathrm{C}$ as a function of applied voltage. The inset is the original SERS spectra showing the corresponding bands. (c) SERS spectra of DMPIC (from the bottom to the top) enhanced by Au NPs in colloidal suspension, $\mathrm{Au} / \mathrm{Co}(\mathrm{OH})_{2}, \mathrm{Au} / \mathrm{Ni}(\mathrm{OH})_{2}$, and $\mathrm{Au} / \mathrm{NiCo} \mathrm{LDH}$, respectively.

the electron-deficient $\mathrm{Au}$ at positive potentials $(0$ to $+0.4 \mathrm{~V})$. Fig. $2 \mathrm{c}$ shows the corresponding SERS spectra in the spectral range from 2100 to $2250 \mathrm{~cm}^{-1}$. The $\mathrm{N} \equiv \mathrm{C}$ stretching on the $\mathrm{Co}(\mathrm{OH})_{2}, \mathrm{Ni}(\mathrm{OH})_{2}$ and NiCo LDH exhibits a red-shift of 8,12 and $14 \mathrm{~cm}^{-1}$, respectively, compared with the SERS band at $2172 \mathrm{~cm}^{-1}$ on $\mathrm{Au}$ NPs in colloidal suspension (Fig. 2c, red curve). According to the band position, the electron transfer to $\mathrm{Au}$ NPs from $\mathrm{Co}(\mathrm{OH})_{2}, \mathrm{Ni}(\mathrm{OH})_{2}$ and NiCo LDH corresponds to an external potential of $-0.19,-0.29$ and $-0.34 \mathrm{~V}$, respectively.

NiCo LDH is a better electron donor for Au NPs compared with $\mathrm{Co}(\mathrm{OH})_{2}$ and $\mathrm{Ni}(\mathrm{OH})_{2}$. This spontaneous electron transfer is further confirmed by XPS analysis. Compared with the pristine NiCo $\mathrm{LDH}$, the binding energy of $\mathrm{Ni}$ and $\mathrm{Co}$ in the $\mathrm{Au} / \mathrm{NiCo}$ LDH is positively shifted by 0.3 and $0.4 \mathrm{eV}$, respectively, suggesting the strong tendency of electron transfer from NiCo LDH to Au NPs (Fig. S8 $\$)^{25}$

\section{Charge transfer promoted electrocatalytic activity}

The electrocatalytic performances of $\mathrm{Au} / \mathrm{NiCo} \mathrm{LDH}, \mathrm{Au} / \mathrm{Ni}(\mathrm{OH})_{2}$ and $\mathrm{Au} / \mathrm{Co}(\mathrm{OH})_{2}$ nanocomposites were evaluated. As shown in Fig. S9, the Au/NiCo LDH exhibits the best performance in the OER among the three Au NP-decorated semiconductors. Compared with the corresponding materials without Au NPs, $\mathrm{Au} / \mathrm{NiCo} \mathrm{LDH}$ also shows the largest improvement of catalytic activity upon the Au NP decoration (Fig. 3a), which can be attributed to the enhanced oxidative reactivity of NiCo LDH after donating electrons to the metal. The same trend was observed in the HER (Fig. 3b): the electron-abundant Au may contribute additional reductive sites for the hydrogen evolution (vide infra). Taken together, the spontaneous charge transfer between $\mathrm{Au}$ and hydroxides promotes the intrinsic electrocatalytic activity of

the materials in both the OER and HER. Because of the highest charge transfer tendency and catalytic activity among the three nanocomposites, we chose Au/NiCo LDH as the plasmonic electrocatalyst for water splitting under visible light irradiation.

\section{Photoelectrocatalytic performance of Au/NiCo LDH for water splitting}

Upon resonant electromagnetic excitation, the plasmonic hot electrons generated by Au NPs were expected to further improve the electrocatalytic performance. The experiment was performed using a typical three-electrode system to investigate the electrocatalytic water splitting under visible light irradiation. As shown in Fig. 4a and b, to reach a current density of $10 \mathrm{~mA}$
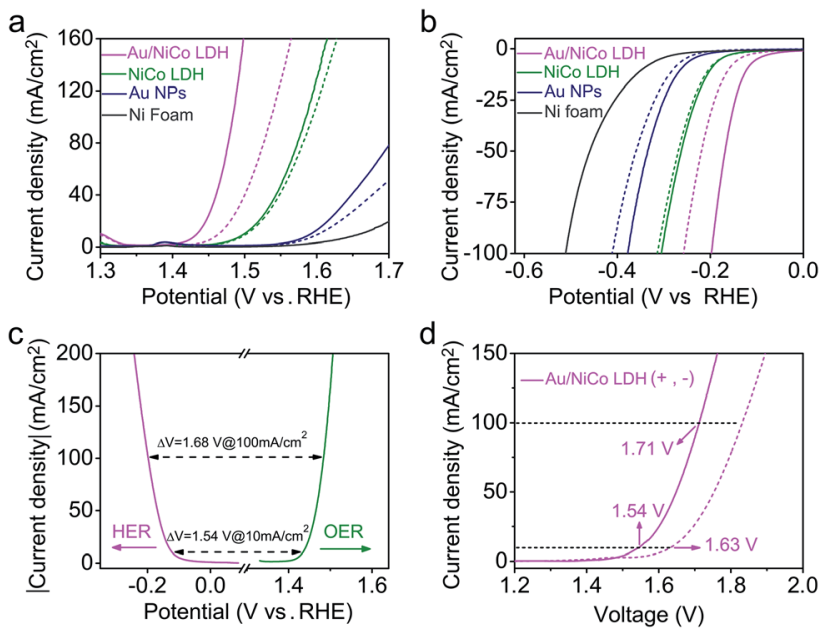

Fig. 4 PEC performance of the prepared catalysts. (a) OER and (b) HER polarization curves of $\mathrm{Au} / \mathrm{NiCo} \mathrm{LDH}, \mathrm{NiCo} L D H, A u$ NPs, and Ni foam measured in $1.0 \mathrm{M} \mathrm{KOH}$ electrolyte with (solid curves) and without (dashed curves) visible light irradiation. (c) Polarization curves of Au/ $\mathrm{NiCO}$ LDH for the HER (pink curve) and OER (green curve) and the voltage difference between the HER and OER at current densities of 10 and $100 \mathrm{~mA} \mathrm{~cm}^{-2}$. (d) Polarization curves for overall water splitting with (solid curve) and without (dashed curve) irradiation on $\mathrm{Au} / \mathrm{NiCO}$ $\mathrm{LDH}$. 
$\mathrm{cm}^{-2}$, the $\mathrm{Au} / \mathrm{NiCo} \mathrm{LDH}$ requires an overpotential of 203 and $119 \mathrm{mV}$ for the OER and HER, respectively (details are shown in Fig. S10 ). Both the Tafel slopes of $42.3 \mathrm{mV} \mathrm{dec}^{-1}$ in the OER and $57.5 \mathrm{mV} \mathrm{dec}^{-1}$ in the HER are much lower than the corresponding values obtained from control experiments by using NiCo LDH or Au NPs. Fig. S11: shows the electrochemical impedance spectra (EIS) and the corresponding equivalent circuit model. It can be obviously seen that the Au/NiCo LDH possesses the lowest charge-transfer resistance under irradiation, suggesting that the plasmonic excitation of Au leads to higher charge transport efficiency in the electrode.

It would be interesting to see the impact of the amount of loaded $\mathrm{Au}$ NPs on the visible light-enhanced electrocatalytic performance. We compared the activity of Au/NiCo LDH with 1.7, 3.3 and $5.1 \mathrm{wt} \% \mathrm{Au}$ content (Fig. S12 $\%$ ). The medium loading amount of Au NPs (3.3 wt\%) exhibits the best activity in both OER and HER experiments. The decrease in activity with more Au NPs is probably due to the aggregation of the NPs when the loading density is too high, which results in the reduced plasmonic activity of the Au/NiCo LDH. Thus, the nanocomposite with $3.3 \mathrm{wt} \% \mathrm{Au}$ NPs was used in all further experiments. In order to investigate the influence of light irradiation on the counter electrode, we performed control experiments in which only the working electrode or counter electrode was under irradiation. As shown in Fig. S13, there is no difference whether the counter electrode is illuminated or not. Besides, the high stability of this Au/NiCo LDH was confirmed by chronopotentiometry curves and high-resolution XPS results (see Fig. S14). Other electrochemical properties of the measured electrodes are summarized in Table S1.\$

Next, the Au/NiCo LDH was used on both the anode and cathode to perform overall water splitting. An applied potential of $1.54 \mathrm{~V}$ is required to reach a current density of $10 \mathrm{~mA} \mathrm{~cm}^{-2}$ under visible light irradiation, which is much lower than the value of $1.63 \mathrm{~V}$ in the dark. Finally, the gaseous products from the overall water splitting on Au/NiCo LDH electrodes were determined using gas chromatography. As illustrated in Fig. S15a, $\$ 0.552 \mathrm{mmol}$ of $\mathrm{O}_{2}$ and $1.107 \mathrm{mmol}$ of $\mathrm{H}_{2}$ are produced after $3 \mathrm{~h}$ of irradiation, corresponding to faradaic efficiencies of $98.63 \%$ (photoanode) and $98.92 \%$ (photocathode), respectively (calculation details are shown in ESI S2\$). The long-term water splitting stability under visible light irradiation was evaluated by a chronopotentiometry method at current densities of 10 and $20 \mathrm{~mA} \mathrm{~cm} \mathrm{~cm}^{-2}$. As shown in Fig. S15b, the voltage of the electrolyzer remains stable during the test. Moreover, the scanning electron microscopic (SEM)/TEM images of $\mathrm{Au} / \mathrm{NiCo} \mathrm{LDH}$ after the stability test in Fig. S16: reveal that the structure of $\mathrm{Au} / \mathrm{NiCo} \mathrm{LDH}$ is well preserved, which indicates that the catalyst has an excellent stability under the real electrocatalytic water splitting reaction conditions. Tables S2-S4\$ compare the performance of Au/NiCo LDH with that of other materials that are among the recently reported best catalysts for the OER, HER and overall water splitting.

\section{Verification of the SPR effect}

Since the plasmonic activity of Au NPs is wavelengthdependent, it is necessary to evaluate the catalytic performance using different light colors. Fig. S17at shows the OER performance of Au/NiCo LDH under 420, 500, 550, 600, and $700 \mathrm{~nm}$ irradiation, respectively. We can see that the $\mathrm{Au} /$ NiCo LDH exhibits the best activity at $550 \mathrm{~nm}$ irradiation. Besides, we measured the photocurrent of Au/NiCo LDH to calculate the incident photon-to-current efficiency (IPCE) at single wavelengths (Fig. S17b末). As shown in Fig. 5a, it's clear that the Au/NiCo LDH exhibits the highest IPCE at $\sim 550 \mathrm{~nm}$, which is also close to the LSPR extinction peak of the Au NPs (Fig. 5a, red curve), further confirming the effect of SPR of Au NPs (details of IPCE are shown in S3†).

However, the non-radiative decay of plasmons not only generates hot electron-hole pairs but also produces heat via coupling with phonons. ${ }^{41}$ Thus it remains unclear whether the hot carriers or the photothermal effect dominates the plasmonpromoted electrocatalytic water splitting. Control experiments in the dark at different temperatures (Fig. S17c indicate that the electrocatalytic activity of Au/NiCo LDH is not significantly enhanced at elevated temperatures of 35 and $45{ }^{\circ} \mathrm{C}$. Fig. 5b compares the current densities and overpotentials for the OER at different temperatures with or without light irradiation. It can be observed that the Au/NiCo LDH under irradiation $\left(25^{\circ} \mathrm{C}\right)$ possesses the largest current density and lowest overpotential. To further verify whether the plasmon effect proceeds via charge separation or thermal enhancement, we isolated the Au NPs with an ultrathin $\mathrm{SiO}_{2}$ shell to prevent the hot electrons from moving to the NiCo LDH. ${ }^{42,43}$ As expected, bare Au decorated NiCo LDH exhibits a much higher PEC activity than ${\mathrm{Au} @ \mathrm{SiO}_{2}}_{2}$ decorated NiCo LDH (Fig. S18\%). Thus, it is the interfacial electron transfer rather than the heat that makes the dominant contribution to the enhanced catalytic activity. ${ }^{44}$ The $i-t$ curve with irradiation switched on and off is shown in Fig. 5c; it can

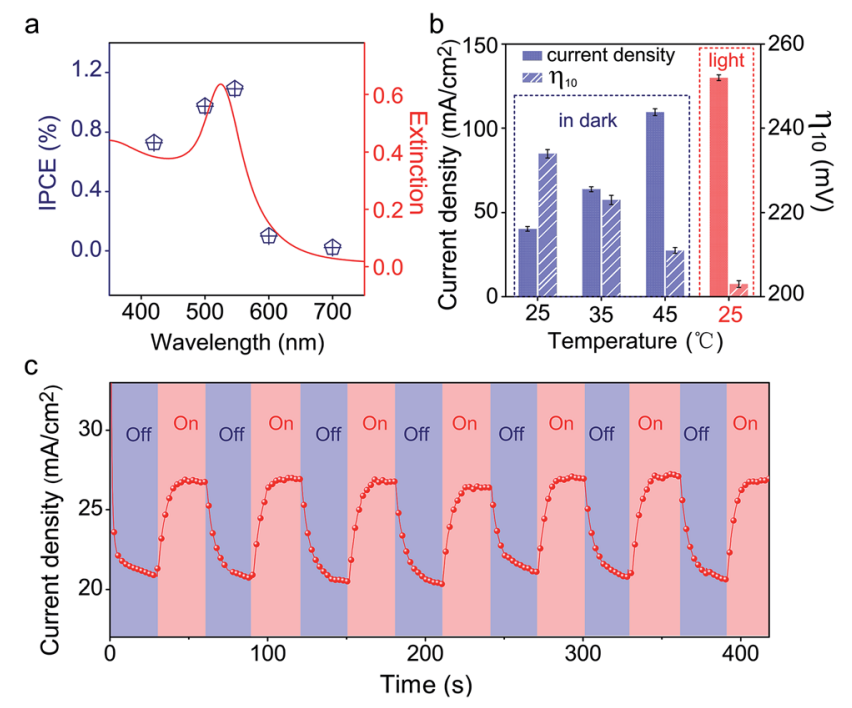

Fig. 5 Verification of the SPR effect. (a) IPCE values of Au/NiCo LDH at different wavelengths (blue points) and the extinction spectrum of $A u$ NPs (red curve). (b) The current densities (solid column) at $1.5 \mathrm{~V}$ and the overpotentials at $10 \mathrm{~mA} \mathrm{~cm}{ }^{-2}$ (striped column) of Au/NiCo LDH under different reaction (OER) temperatures with or without light irradiation. (c) Chronoamperometric $i-t$ curve of the Au/NiCo LDH under chopped irradiation. 
be observed clearly that switching off the light restricts the oxygen evolution.

In order to confirm the real catalytic surface on $\mathrm{Au} / \mathrm{NiCo} \mathrm{LDH}$ during the plasmon-promoted electrocatalytic water splitting, a thiol compound, thiophenol (TP), was used to coat the Au surface through strong covalent $\mathrm{Au}-\mathrm{S}$ bonds (Fig. 6); thus the $\mathrm{Au}$ surface of the $\mathrm{Au} / \mathrm{NiCo} \mathrm{LDH}$ is selectively blocked for catalysis. ${ }^{45}$ As evidenced in Fig. S19, $\$$ after incubation with TP, Au NPs exhibit a significantly decreased activity but NiCo LDH shows no obviously change, in both the HER and OER. The overpotentials of the different catalysts for the HER and OER are presented in Fig. 6a and c, respectively. In the HER, the remarkably increased overpotential value of $\mathrm{Au} / \mathrm{NiCo} \mathrm{LDH}$ after the TP coating suggests that the plasmon-promoted water reduction mainly occurs on the $\mathrm{Au}$ surface (vide supra). In contrast, the overpotential value does not change significantly in the OER, indicating that NiCo LDH is the main catalytic material for oxygen evolution.

Based on the above results, a proposed mechanism of the plasmon-promoted electrocatalysis is illustrated in Fig. 7. The electron transfer from NiCo $\mathrm{LDH}$ to Au enhances the intrinsic electrocatalytic activity of the nanocomposite. For NiCo $\mathrm{LDH}$, the conduction band (CB) minimum and the valence band (VB) maximum are estimated to be -4.21 and $-5.77 \mathrm{eV}$ (Fig. S20 $\$$ ), respectively. ${ }^{46}$ Under resonant light irradiation, hot electrons are generated on the Au NPs via non-radiative decay of the surface plasmons. ${ }^{47}$ On the cathode side (Fig. 7, left), hot electrons on the Au surface of $\mathrm{Au} / \mathrm{NiCo} \mathrm{LDH}$ are utilized more easily since the plasmon-generated holes are compensated for by both

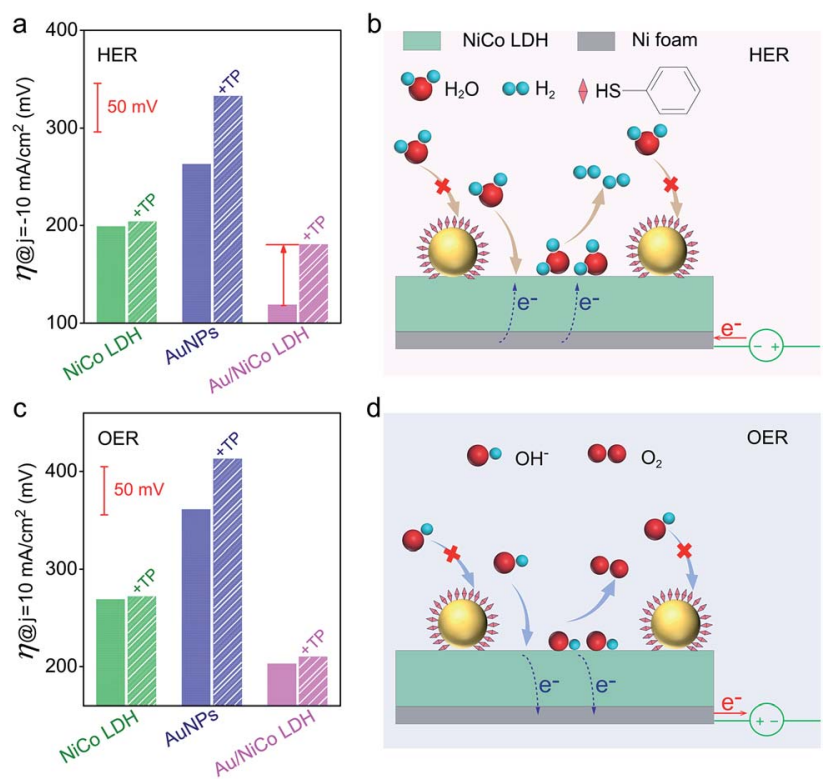

Fig. 6 Determination of real catalytic surfaces. (a) Overpotentials of $\mathrm{NiCo} \mathrm{LDH}, \mathrm{Au}$ NPs and Au/NiCo LDH with (striped column) and without (solid column) TP monolayer coating on the Au NPs, under light irradiation toward the HER. (b) Schematic illustration of water reduction on the Au@TP/NiCo LDH. (c) Overpotentials of NiCo LDH, $\mathrm{Au}$ NPs and Au/NiCo LDH with (striped column) and without (solid column) TP coating under light irradiation toward the OER. (d) Schematic illustration of $\mathrm{OH}^{-}$oxidation on the Au@TP/NiCo LDH.

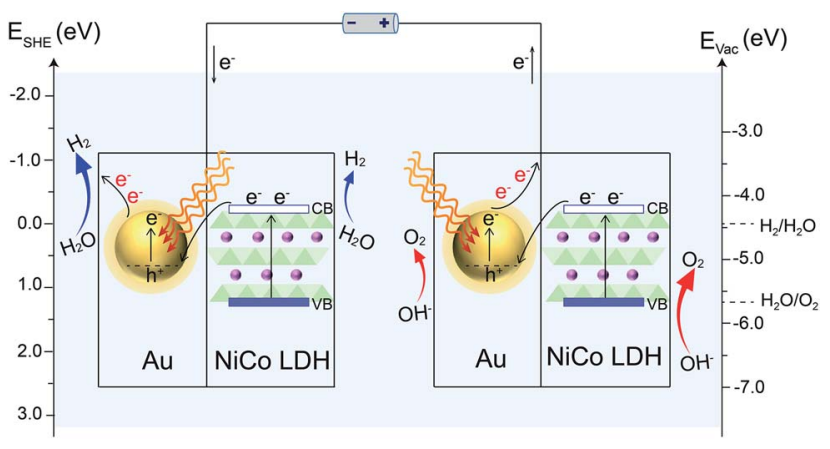

Fig. 7 Proposed reaction mechanism. Schematic illustration of the plasmon-promoted electrocatalytic water splitting on Au/NiCo LDH under visible light irradiation.

the electrons from the NiCo LDH and foreign electrons from the anode. As demonstrated in Fig. 6a, the main catalytic surface for the plasmon-enhanced HER is Au rather than NiCo LDH. Under resonant irradiation, the $\mathrm{Au} / \mathrm{NiCo} \mathrm{LDH}$ exhibits a Tafel slope of $57.5 \mathrm{mV} \mathrm{dec}{ }^{-1}$ (Fig. S10c ), indicating the Volmer-Heyrovsky path (Scheme S1 $\$$ ) in which the hot electrons participate in the reduction of water and promote the evolution of hydrogen. ${ }^{\mathbf{4 8 , 4 9}}$ On the anode side (Fig. 7, right), the hot electrons are further transferred to the cathode upon the applied potential. ${ }^{12}$ Since $\mathrm{Au}$ is not a good OER catalyst compared with NiCo LDH (as shown in Fig. 4a), electrochemical water oxidation on the $\mathrm{Au}$ surface of $\mathrm{Au} / \mathrm{NiCo} \mathrm{LDH}$ by the plasmon-generated holes is subordinate to that on the NiCo LDH surface. Meanwhile, beside the spontaneous charge transfer from NiCo $\mathrm{LDH}$ to $\mathrm{Au}$, the plasmon-generated holes are effective electron trappers to capture electrons from NiCo LDH, remarkably enhancing the electron transfer from NiCo LDH to Au NPs for the formation of oxidative NiCo LDH. Then, the oxidative NiCo LDH species provide active sites to extract electrons from $\mathrm{OH}^{-}$, facilitating the formation and subsequent deprotonation of the key intermediates and thus promoting the OER catalysis (Scheme S1†). ${ }^{50,51}$

\section{Conclusions}

In summary, we presented a mechanistic study of plasmonpromoted electrocatalytic water splitting. Via integration of $\mathrm{Au}$ NPs and conventional semiconductor electrocatalysts including $\mathrm{Co}(\mathrm{OH})_{2}, \mathrm{Ni}(\mathrm{OH})_{2}$ and NiCo LDH, the catalytic performance was significantly enhanced under visible light irradiation. To investigate the interfacial charge transfer direction in the metal-semiconductor nanocomposites, SERS was employed to measure the molecular vibrational band shifts caused by the electronic property changes on the Au surface. We found that there is a simultaneous electron transfer from the semiconductor to the metal. As expected, Au/NiCo LDH with the highest electron transfer tendency among the three exhibited the best electrocatalytic performance, in both reactions with and without visible light irradiation. It has been confirmed by wavelength-dependent experiments that the LSPR effect is responsible for the enhanced activity under irradiation. To 
disentangle the contributions from hot carriers and heat, we performed temperature-dependent and ultrathin shell-isolation controls which demonstrated the dominant role of hot carriers. Finally, the real catalytic sites of the plasmonic electrocatalysis were determined by selective coating of the Au surface on the nanocomposite with a thiol monolayer. After coating, the catalytic activity for water reduction decreased remarkably while no obvious change was found in the oxidation half reaction, indicating that the HER and OER mainly took place on $\mathrm{Au}$ and semiconductor (NiCo LDH) surfaces, respectively. This mechanistic study highlights the important synergistic effect between the light harvester (i.e. the plasmonic NPs) and the conventional electrocatalyst for visible light-enhanced water splitting.

\section{Conflicts of interest}

There are no conflicts to declare.

\section{Acknowledgements}

We acknowledge the financial support from the National Natural Science Foundation of China (21775074, 51601098 \& 21861132016), the Natural Science Foundation of Tianjin (17JCQNJC05400), and the National Key R\&D Program (2017YFA0206702 \& 2016YFB0901502).

\section{Notes and references}

1 S. Mukherjee, L. Zhou, A. M. Goodman, N. Large, C. AyalaOrozco, Y. Zhang, P. Nordlander and N. J. Halas, J. Am. Chem. Soc., 2014, 136, 64-67.

2 B. Foerster, A. Joplin, K. Kaefer, S. Celiksoy, S. Link and C. Sonnichsen, ACS Nano, 2017, 11, 2886-2893.

3 C. Hu, T. Peng, X. Hu, Y. Nie, X. Zhou, J. Qu and H. He, J. Am. Chem. Soc., 2010, 132, 857-862.

4 E. C. Dreaden, A. M. Alkilany, X. Huang, C. J. Murphy and M. A. El-Sayed, Chem. Soc. Rev., 2012, 41, 2740-2779.

5 Y. Kim, J. G. Smith and P. K. Jain, Nat. Chem., 2018, 10, 763769.

6 J. Xu, W.-M. Yang, S.-J. Huang, H. Yin, H. Zhang, P. Radjenovic, Z.-L. Yang, Z.-Q. Tian and J.-F. Li, Nano Energy, 2018, 49, 363-371.

7 H. Jia, A. Du, H. Zhang, J. Yang, R. Jiang, J. Wang and C. Y. Zhang, J. Am. Chem. Soc., 2019, 141, 5083-5086.

8 H. Zhang, T. Wang, J. Wang, H. Liu, T. D. Dao, M. Li, G. Liu, X. Meng, K. Chang, L. Shi, T. Nagao and J. Ye, Adv. Mater., 2016, 28, 3703-3710.

9 J. Yang, Y. Guo, R. Jiang, F. Qin, H. Zhang, W. Lu, J. Wang and J. C. Yu, J. Am. Chem. Soc., 2018, 140, 8497-8508.

10 S. Mubeen, J. Lee, N. Singh, S. Kramer, G. D. Stucky and M. Moskovits, Nat. Nanotechnol., 2013, 8, 247-251.

11 J. Homola, Chem. Rev., 2008, 108, 462-493.

12 J. Xu, P. Gu, D. J. S. Birch and Y. Chen, Adv. Funct. Mater., 2018, 28, 1801573.

13 S. Atta, A. M. Pennington, F. E. Celik and L. Fabris, Chem, 2018, 4, 2140-2153.
14 R. Shi, Y. Cao, Y. Bao, Y. Zhao, G. I. N. Waterhouse, Z. Fang, L. Z. Wu, C. H. Tung, Y. Yin and T. Zhang, Adv. Mater., 2017, 29, 1700803.

15 B. S. Hoener, S. R. Kirchner, T. S. Heiderscheit, S. S. E. Collins, W.-S. Chang, S. Link and C. F. Landes, Chem, 2018, 4, 1560-1585.

16 J. Lee, S. Mubeen, X. Ji, G. D. Stucky and M. Moskovits, Nano Lett., 2012, 12, 5014-5019.

17 L. Yu, J. F. Yang, B. Y. Guan, Y. Lu and X. W. D. Lou, Angew. Chem., Int. Ed., 2018, 57, 172-176.

18 J. Luo, J. H. Im, M. T. Mayer, M. Schreier, M. K. Nazeeruddin, N. G. Park, S. D. Tilley, H. J. Fan and M. Gratzel, Science, 2014, 345, 1593-1596.

19 Y. Chao, P. Zhou, N. Li, J. Lai, Y. Yang, Y. Zhang, Y. Tang, W. Yang, Y. Du, D. Su, Y. Tan and S. Guo, Adv. Mater., 2019, 31, e1807226.

20 Z. Zhao, H. Wu, H. He, X. Xu and Y. Jin, Adv. Funct. Mater., 2014, 24, 4698-4705.

21 S. Linic, P. Christopher and D. B. Ingram, Nat. Mater., 2011, 10, 911-921.

22 N. Zhang, C. Han, X. Fu and Y.-J. Xu, Chem, 2018, 4, 18321861.

23 P. Christopher, H. Xin and S. Linic, Nat. Chem., 2011, 3, 467472.

24 Y. Shi, J. Wang, C. Wang, T. T. Zhai, W. J. Bao, J. J. Xu, X. H. Xia and H. Y. Chen, J. Am. Chem. Soc., 2015, 137, 7365-7370.

25 G. Liu, P. Li, G. Zhao, X. Wang, J. Kong, H. Liu, H. Zhang, K. Chang, X. Meng, T. Kako and J. Ye, J. Am. Chem. Soc., 2016, 138, 9128-9136.

26 W. Xie, R. Grzeschik and S. Schlücker, Angew. Chem., Int. Ed., 2016, 55, 13729-13733.

27 Y. L. Li, Y. F. Hu, F. X. Shi, H. X. Li, W. Xie and J. Chen, Angew. Chem., Int. Ed., 2019, 58, 9049-9053.

28 O. Diaz-Morales, D. Ferrus-Suspedra and M. T. M. Koper, Chem. Sci., 2016, 7, 2639-2645.

29 J. Ye, F. Wen, H. Sobhani, J. B. Lassiter, P. Van Dorpe, P. Nordlander and N. J. Halas, Nano Lett., 2012, 12, 16601667.

30 H. Zhang, X. G. Zhang, J. Wei, C. Wang, S. Chen, H. L. Sun, Y. H. Wang, B. H. Chen, Z. L. Yang, D. Y. Wu, J. F. Li and Z. Q. Tian, J. Am. Chem. Soc., 2017, 139, 10339-10346.

31 H. Zhang, X. Li, A. Hähnel, V. Naumann, C. Lin, S. Azimi, S. L. Schweizer, A. W. Maijenburg and R. B. Wehrspohn, Adv. Funct. Mater., 2018, 28, 1706847.

32 Z. Zheng, W. Xie, M. Li, Y. H. Ng, D.-W. Wang, Y. Dai, B. Huang and R. Amal, Nano Energy, 2017, 41, 233-242.

33 S. Yu, A. J. Wilson, J. Heo and P. K. Jain, Nano Lett., 2018, 18, 2189-2194.

34 R. Gómez, J. Solla-Gullón, J. M. Pérez and A. Aldaz, J. Raman Spectrosc., 2005, 36, 613-622.

35 D. Zha, Y. Fu, L. Zhang, J. Zhu and X. Wang, J. Power Sources, 2018, 378, 31-39.

36 B. D. Busbee, S. O. Obare and C. J. Murphy, Adv. Mater., 2003, 15, 414-416.

37 Y. J. Li, W. J. Huang and S. G. Sun, Angew. Chem., Int. Ed., 2006, 45, 2537-2539. 
38 H. S. Su, X. G. Zhang, J. J. Sun, X. Jin, D. Y. Wu, X. B. Lian, J. H. Zhong and B. Ren, Angew. Chem., Int. Ed., 2018, 57, 13177-13181.

39 Y.-H. Wang, M.-M. Liang, Y.-J. Zhang, S. Chen, P. Radjenovic, H. Zhang, Z.-L. Yang, X.-S. Zhou, Z.-Q. Tian and J.-F. Li, Angew. Chem., Int. Ed., 2018, 57, 11257-11261.

40 S. Hu, B. J. Liu, J. M. Feng, C. Zong, K. Q. Lin, X. Wang, D. Y. Wu and B. Ren, J. Am. Chem. Soc., 2018, 140, 1368013686.

41 L. Zhou, D. F. Swearer, C. Zhang, H. Robatjazi, H. Zhao, L. Henderson, L. Dong, P. Christopher, E. A. Carter, P. Nordlander and N. J. Halas, Science, 2018, 362, 69-72.

42 C. Li, P. Wang, Y. Tian, X. Xu, H. Hou, M. Wang, G. Qi and Y. Jin, ACS Catal., 2017, 7, 5391-5398.

43 C. Li, D. Cahen, P. Wang, H. Li, J. Zhang and Y. Jin, iScience, 2018, 8, 213-221.

44 L. Mascaretti, A. Dutta, S. Kment, V. M. Shalaev, A. Boltasseva, R. Zboril and A. Naldoni, Adv. Mater., 2019, e1805513.
45 W. Xie, B. Walkenfort and S. Schlücker, J. Am. Chem. Soc., 2013, 135, 1657-1660.

46 Y. Zhao, X. Jia, G. I. N. Waterhouse, L.-Z. Wu, C.-H. Tung, D. O'Hare and T. Zhang, Adv. Energy Mater., 2016, 6, 1501974.

47 H. X. Zhang, Y. Li, M. Y. Li, H. Zhang and J. Zhang, Nanoscale, 2018, 10, 2236-2241.

48 Q. Q. Chen, C. C. Hou, C. J. Wang, X. Yang, R. Shi and Y. Chen, Chem. Commun., 2018, 54, 6400-6403.

49 L. Yu, I. K. Mishra, Y. Xie, H. Zhou, J. Sun, J. Zhou, Y. Ni, D. Luo, F. Yu, Y. Yu, S. Chen and Z. Ren, Nano Energy, 2018, 53, 492-500.

50 J. Zhang, J. Liu, L. Xi, Y. Yu, N. Chen, S. Sun, W. Wang, K. M. Lange and B. Zhang, J. Am. Chem. Soc., 2018, 140, 3876-3879.

51 M. S. Burke, M. G. Kast, L. Trotochaud, A. M. Smith and S. W. Boettcher, J. Am. Chem. Soc., 2015, 137, 3638-3648. 